\title{
PESQUISA CIENTÍFICA EM CONTABILIDADE ENTRE 1990 E 2003
}

RESUMO

Este artigo analisa a distribuição, as características metodológicas, a evolução e a temática das publicações científicas em contabilidade, bem como a produção de seus autores. 0 período pesquisado foi entre 1990 e 2003, nas revistas nacionais classificadas com conceito "A" pela Capes. 0 método utilizado foi 0 da pesquisa descritiva. Do total de 2.037 artigos publicados nesse período, 60 foram identificados como de contabilidade. A análise indicou que as instituições com maior número de publicações, em ordem decrescente, foram a USP, FGV-SP, FGV-RJ e UFRGS. São Paulo foi o estado com o maior número de artigos publicados. A análise bibliométrica, utilizando a Lei de Lotka, revel ou a produtividade dos autores no referido período. Os resultados foram comparados com os de alguns autores, constatando-se que o número de autores com uma única publicação é maior do que o indicado pela literatura.

\section{Ricardo Lopes Cardoso}

PUC-Campinas

\section{Octávio Ribeiro de Mendonça Neto} UMESP

\section{Edson Luiz Riccio}

USP

\section{Marici Cristine Gramacho Sakata}

USP

\begin{abstract}
Thearticleanalyzes distribution, methodology, evolution, and topics in accounting scientific publications, as well as authors' contributions. The period covered was 1990 through 2003, in Brazilian journals with a Capes ranking of A. The methodology was descriptive research; using a structured questionnaire. Out of the 2,037 articles published in the period under study, 60 were found to be on accounting. D escriptive analysis showed that institutions with highest number of publications were ranked as follows: USP, FGV-SP, FGV-RJ and U FRGS. São Paulo state stands at the top for number of published articles. Bibliometric analysis using Lotka Law showed authors' productivity in that period. Results also showed that the number of authors with one publication only is higher than data available in the literature.
\end{abstract}

PALAVRAS-CHAVE Contabilidade, produção científica, periódicos nacionais, cientometria, bibliometria.

KEYWORDS Accounting, scientific publications, Brazilian journals, scientometrics, bibliometrics. 


\section{INTRODUÇÃO}

0 processo de avaliação da ciência não é recente no meio acadêmico. M apear e conhecer trabalhos acadêmicos publicados em determinada área por meio de revisões sistemáticas é uma das formas de possibilitar a avaliação e a reflexão desses trabalhos e da área em questão. Embora uma das principais razões de sua crescente aplicação se deva à necessidade de direcionar recursos de instituições educacionais e governamentais para pesquisa, tal análise permite detectar indicadores, tendências e vieses de cada área.

As revisões são em geral feitas por técnicas tradicionais no meio acadêmico, como a análise bibliométrica. Como parte importante do processo de compreensão da ciência, a anál ise bibliométrica deve ser acompanhada de pesquisas qualitativas para que os seus resultados possam ser interpretados em sua abrangência.

Estudos dessa natureza foram desenvolvidos em diferentes áreas no Brasil, com destaque para o marketing (Vieira, 1998), organizações (Machado-da-Silva, Cunha e Amboni, 1990; Vergara, 2000), recursos humanos (Tonelli et al., 2003; Caldas e Tinoco, 2004), gerência de operações (Arkader, 2003), finanças (Leal, Oliveira e Soluri, 2003), contabilidade (Riccio, Carastan e Sakata, 1999; Frezatti, 2000; M artins, 2002; Oliveira, 2002) e sistemas de informação (Hoppen e M eirelles, 2005). No âmbito internacional se destacam na área de contabilidade os trabal hos de Bricker (1989), Chung, Cox e Pak (1992), Zeff (1996), e Shields (1997). Esse tipo de estudo é classificado na área da bibliometria, e tem como grandes desafios a delimitação da contabilidade no âmbito da Administração e as respectivas classificações metodológicas.

Este artigo se propõe preencher essa fal ta de informações sobre a produção científica em contabilidade, saber qual foi o enfoque adotado e quem são os pesquisadores. Na primeira parte é discutido o emprego da bibliometria na produção acadêmica no campo da contabilidade. $\mathrm{N}$ a segunda seção são apresentados os aspectos metodológicos da pesquisa, tais como o método, a população e a amostra, e de que forma serão feitas as análises. $\mathrm{N}$ a terceira seção são discutidos os resultados da pesquisa, ressaltando aspectos da produção acadêmica em contabilidade, como a produtividade, distribuição geográfica, temas tratados, metodologias empregadas pelos autores e comparação da produtividade dos autores. A última parte contém as considerações finais, limitações da pesquisa e sugestões para futuros estudos.

\section{ESTUDO DA BIBLIOMETRIA E SUA APLICAÇÃO NO CAMPO DA CONTABILIDADE}

De acordo com Macias-Chapula (1998), a bibliometria estuda os aspectos quantitativos da produção, da disseminação e do uso da informação registrada. Foi usada pela primeira vez por Pritchard, em 1969. Atualmente, a bibliometria desenvolve padrões e model os matemáticos para medir os processos, usando seus resultados para elaborar previsões e apoiar tomadas de decisão.

Os teóricos e pesquisadores da bibliometria desenvolveram e criaram "Ieis" específicas para a análise da produção científica. Para Chen, Chong e Tong (1994), as três leis de distribuição bibliométrica mais conhecidas nessa disciplina são a Lei de Lotka, a Lei de Zipf e a Lei de Bradford. Vanti (2002) define cada uma das leis da seguinte forma: a Lei de Lotka, ou Lei do Quadrado Inverso, refere-se à medição da produtividade dos autores mediante um modelo de distribuição de tamanho-freqüência dos diversos autores em um conjunto de documentos. A Lei de Zipf, ou Lei do Mínimo Esforço, consiste em medir a freqüência do aparecimento das palavras em vários textos, gerando uma lista ordenada de termos de uma determinada disciplina ou assunto. A Lei de Bradford, ou Lei de Dispersão, permite, mediante a medição da produtividade das revistas, estabelecer o núcleo e as áreas de dispersão sobre um determinado assunto em um mesmo conjunto de revistas.

As leis bibliométricas têm sido utilizadas em diversas pesquisas e para diferentes fins, conforme os estudos de Leydesdorff (2001), Verbeek et al. (2002), Da Silva (2001) e Souza (2003). Para analisar a existência de uma disciplina ou área de estudo, autores como Pilkington e Liston-Heyes (1999) utilizaram as distribuições bibliométricas. Estudos aplicados utilizando o enfoque bibliométrico foram realizados por autores como Findlay e Sparks (2002) na área de varejo, e por G arcia-Castrillo, M ontañés e Sanz-Gracia (2002) na área de Economia, ambos analisando periódicos internacionais. No campo da gestão, artigos como o de Park e Gordon (1996) sobre gestão estratégica, e de Spell (1999) sobre modismos em gestão, e trabal hos em áreas gerais da Administração - como os de Carson et al. (1999), Mcmillan e Hamilton (2000), e M cmillan e Hicks (2001) - foram identificados como tentativas de medir o desenvolvimento da área de conhecimento em questão.

Quanto à aplicação desse instrumento na anál ise de publicações em contabilidade e finanças, é importan- 
te ressaltar o trabalho de Chung e Cox (1990), que buscou medir a regularidade da literatura em finanças e sua relação com as autorias dos artigos. Em 1992, Chung, Cox e Pak examinaram a existência de regularidade nas publicações de pesquisadores em contabilidade. Bricker (1989) e Zeff (1996) também estudam essa questão, e há o destaque ao artigo de Shields (1997), que analisa, na área de contabilidade gerencial, vários aspectos sobre as publicações em periódicos internacionais de contabilidade. Mais recentemente há o estudo de Otchere (2003), que analisa em periódicos internacionais aspectos da produtividade dos autores, bem como aspectos das citações em contabilidade. No Brasil, a bibliométrica aplicada à área de finanças é encontrada no trabalho de Leal, Oliveira e Soluri (2003), que realizam levantamento histórico de várias publicações na área no Brasil e analisam os resultados constatados com a aplicação da Lei de Lotka. 0 presente artigo seguirá esse caminho, ao realizar uma análise comparativa da produtividade dos autores na área de contabilidade por meio da utilização da Lei de Lotka.

\section{ASPECTOS METODOLÓGICOS}

\section{Método de pesquisa}

0 método utilizado foi a pesquisa descritiva. Esse tipo de pesquisa visa conhecer um fenômeno sem modificá-lo, a fim de entender o objeto de interesse em um determinado espaço e tempo (Selltiz, Cook e Wrightsman, 1987).

Foram selecionadas as revistas para identificar os artigos relacionados à área da contabilidade (veja a Tabela 1). Em uma segunda etapa foram analisados os abstracts para a identificação da temática, dos paradigmas de pesquisa utilizados, da área de negócios abrangida, dos autores e instituições dos autores, bem como a leitura da introdução, dos aspectos metodológicos e das conclusões quando da necessidade de informações mais detal hadas para o desenvolvimento do estudo. Todo o processo de classificação dos dados foi realizado por pelo menos dois dos quatro pesquisadores, objetivando um melhor ajuste de definições e redução das incertezas inerentes a esse tipo de classificação.

\section{Instrumento e processo de validade}

0 instrumento utilizado para a análise dos resumos foi um roteiro estruturado, tendo como base os seguintes itens: título, autor(es), instituição(ões), tema, breve resumo, aspectos metodológicos e setor econômico do artigo. Após a estruturação, testou-se o instrumento numa revista, e, posteriormente, foram introduzidas algumas melhorias no processo de análise, tais como a revisão das tipologias metodológicas utilizadas e ajustes nas classificações temáticas.

\section{População e amostra}

Tomando como referências a base de dados da Capes (Coordenação de Aperfeiçoamento de Pessoal de Nível Superior), SciELO (Scientific Electronic Library Online), e IBICT (Instituto Brasileiro de Informação em Ciência e Tecnologia), o número de revistas científicas nacionais e internacionais nas diversas áreas do conhecimento em 2004 representa um universo de artigos científicos cuja quantidade dificulta a análise. Para esta pesquisa, o critério de inclusão das revistas na amostra é dado pelos seus enquadramentos em categorias indicativas da qualidade " $A$ ", obtida no site da C apes em setembro de 2003. 0 sistema Q ualis, concebido pela Capes, classifica os veículos de divulgação de produção intelectual utilizados por docentes e alunos para fins de avaliação dos programas de pósgraduação em contabilidade no Brasil. As categorias indicativas da qualidade são $A, B$ ou $C$, e seus âmbitos de circulação são local, nacional ou internacional. É importante frisar que, para fins de avaliação, a contabilidade está inserida no campo da Administração.

Da lista de revistas disponíveis, obteve-se acesso a todos os exemplares do período entre 1990 e 2003 das revistas que mantinham relação direta e indireta com a área de contabilidade em suas linhas editoriais, totalizando seis revistas nível "A": PPE-Pesquisa e Planejamento E conômico (IPEA), RAC-Revista de Administração Contemporânea, RBE-Revista Brasileira de Economia (FGV-RJ), RAP-Revista de Administração Pública (FGV-RJ), RAE-Revista de Administração de Empresas (FGV-SP), RAUSP-Revista de Administração (USP). Embora também classificadas com conceito " $A$ ", não foram incluídas a revista Pesquisa Operacional, da Sobrapo (Sociedade Brasileira de Pesquisa O peracional) e a Revista de Administração da UFBA, devido a problemas de acesso aos exemplares.

Das 269 edições dos periódicos científicos examinados, foram encontrados 2.037 artigos publicados em Administração e Economia. A análise identificou um total de 60 artigos de contabilidade, correspondendo a $2,95 \%$ do total, o que revela um baixo volume de publicações dos pesquisadores da área contábil nos periódicos analisados. 


\section{Classificações temáticas}

Para a classificação dos temas abordados nos artigos se optou pela utilização do DDC (Classificação Decimal de Dewey, 1996), tópico de contabilidade (657), conforme adaptado por Riccio, Carastan e Sakata (1999). Tal adaptação adiciona ao DDC os tópicos propostos pelaAAA (American Accounting Association), pela EAA (European Accounting Association) e pelas principais universidades dos Estados Unidos e da Europa.

\section{Análise da metodologia de pesquisa}

A análise da metodologia utilizada pelos autores em seus artigos está baseada nos conceitos apresentados por Denzin e Lincoln (2000). Os autores dividem a pesquisa da seguinte forma: fase 1, o pesquisador; fase 2, paradigma e perspectiva teórica; fase 3, estratégias de pesquisa; fase 4, 0 modelo de coleta e análise de dados; fase 5, apresentação e interpretação dos resultados. 0 modelo utilizado neste trabal ho enfoca as fases 2, 3 e 4 da pesquisa, classificando-as respectivamente em níveis 1, 2 e 3. Para cada nível, o modelo de análise da metodologia considerou os seguintes paradigmas, estratégias e métodos de análise:

- nível 1 - paradigmas: teórico, descritivo, exploratório, explicativo, histórico, causal e outros;

- nível 2 - estratégia: estudo de caso, pesquisa de campo de tipo empírico-descritiva, observação, experimentos de laboratório, bibliográfica e outras;

- nível 3 - análise: interpretação, crítica, expositiva, comprobatória, estatística e outras.

\section{Análise da produtividade dos autores}

Leal, Oliveira e Soluri (2003) utilizam a Lei de Lotka para a análise da produtividade dos autores brasileiros na área de finanças, segundo a qual o número de autores que publica $\mathrm{n}$ artigos é dado por:

$$
\alpha_{n}=\alpha_{1} \cdot \frac{1}{n^{2}}
$$

Sendo:

$\alpha_{1}=$ número de autores que publicam um único artigo;

$\alpha_{n}=$ número de autores que publicam $n$ artigos;

$\mathrm{n}=$ número de artigos.

Os autores observam ainda que, ao pesquisar publicações em química e física, Lotka constatou que o número de autores que publicam uma única vez corresponde a $60 \%$ da amostra. Os autores referem também o trabalho de Chung e Cox (1990), que indica que o número de autores, segundo a Lei de Lotka, é de
$60,8 \%$ do total da amostra, e que o número de autores com duas publicações corresponde a $15,2 \%$ (ou $1 / 4$ de $60,8 \%)$.

No presente trabal ho será utilizada a mesma metodologia, o que possibilitará uma análise comparativa entre os resultados obtidos. Da mesma forma que Chung, Cox e Pak (1992), e Leal, Oliveira e Soluri (2003), será utilizada a Lei de Lotka genérica, expressa por:

$$
\alpha_{n}=\alpha_{1} \cdot \frac{1}{n^{c}}
$$

0 coeficiente $c$ é estimado de acordo com a amostra. Um coeficiente estimado c maior do que 2 - que é o utilizado pela Lei Lotka - significa que o número de autores com uma única publicação é maior do que o estabelecido pela referida lei. 0 coeficiente c foi estimado pela técnica de regressão linear, seguin do o que foi feito pelos autores dos estudos citados anteriormente. 0 modelo é dado por:

$$
\operatorname{Ln}\left(\frac{\alpha_{n}}{\alpha_{1}}\right)=-\operatorname{c} \operatorname{Ln}(n)
$$

\section{RESULTADOS DA PESQUISA}

Os seis periódicos analisados abrangem o período de 1990 a 2003, com exceção da RAC, cuja edição foi iniciada após 1997 (veja a Tabela 1). Do total de 295 exemplares, não foram localizados 26 , que não constavam no acervo das bibliotecas consultadas, o que resulta em 269 o número de revistas analisadas. Nessas revistas há um total de 2.037 artigos, e utilizando-se os critérios adotados para a seleção, 60 desses artigos foram considerados como pertencentes à área de contabilidade, o que representa $2,95 \%$ do total. Para a classificação dos assuntos de contabilidade foi utilizada a leitura detalhada do resumo e, no caso de dúvida, a leitura completa do artigo. Simultaneamente a esse processo, houve discussões com dois membros da equipe de pesquisadores para reduzir as dúvidas de classificação.

A análise por periódico revela que a RAE e a RAUSP são as duas revistas com o maior número de artigos publicados, com 21 e 20 respectivamente, do total de 60 trabalhos. 0 número médio de publicações de contabilidade é de 4,3 artigos por ano, tendo como grandes pontos de produção os anos de 1997 e 2001, com 8 e 9 artigos respectivamente. $\mathrm{Na}$ Tabela 2 pode se constatar 0 aumento da produção acadêmica a partir 
de 1997, o que sugere que pode haver um maior número de artigos publicados nos próximos anos.

Para anal isar a distribuição geográfica dos trabalhos, o critério utilizado para os artigos escritos por mais de um autor foi o de atribuir a cada um a fração correspondente. Por exemplo, no caso de dois autores é atribuído o valor de 0,5 para cada um; para três autores o valor é de 0,33. Dessa forma, pode se observar que a maior parte da produção está concentrada em São Paulo (51,12\% do total). Os autores internacionais representam $8,05 \%$ da produção total. Esses números se diferenciam de outras áreas do conhecimento. Por exemplo, segundo Leal, Oliveira e Soluri (2003), a área de finanças apresenta uma concentração de $67,94 \%$ de artigos de autoria de pesquisadores vinculados a instituições sediadas no estado do Rio de Janeiro. A segunda maior produção na área de contabilidade está concentrada no Rio de Janeiro, com $20 \%$ do total, seguida pelo Rio Grande do Sul, com 10,83\%.

$\mathrm{Na}$ Tabela 3 são classificados os temas da contabilidade abordados pelos artigos analisados. Ganha destaque o tema contabilidade gerencial, que, juntamente com contabilidade de custos e orçamento, correspondem a $53,3 \%$ da produção analisada. 0 terceiro tema mais abordado é o de contabilidade e mercados de capital, que representa $16,7 \%$ do total. Os artigos de contabilidade pública representam
$8,3 \%$ do total e estão concentrados nas revistas PPE e RAP, indicando adequação à linha editorial das referidas publicações.

\section{Análise da metodologia de pesquisa}

A análise da metodologia utilizada pelos autores possui limitações inerentes ao seu processo subjetivo, tanto sob o aspecto das abordagens teóricas utilizadas como nas suas classificações propriamente ditas. Os resultados obtidos são apresentados a seguir.

Observa-se que os totais dos resultados referentes aos níveis 2 e 3 superam o número total de artigos. Isso se deve ao fato de que alguns autores utilizaram mais de uma estratégia de pesquisa ou mais de um método de análise de resultados em um mesmo artigo.

0 termo "paradigma" neste trabal ho significa o arcabouço teórico e filosófico da disciplina ou escola científica na qual teorias, leis e generalizações, bem como os experimentos que as sustentam, são formuladas. Da totalidade dos artigos analisados, os resultados do nível 1 , referente ao paradigma utilizado, foram: descritivo, 41,7\% ( 25 artigos); explicativo, 28,3\% (17 artigos); exploratório, 18,3\% (11 artigos); teórico, 6,7\% (4 artigos); e causal, $5 \%$ ( 3 artigos). N ão foi encontrada a utilização do paradigma histórico na amostra.

Percebe-se que na área de contabilidade a abordagem descritiva é a predominante, com $41,7 \%$. 0 objetivo do

Tabela 1 - Participação dos artigos de contabilidade em relação ao total de artigos publicados.

\begin{tabular}{|c|c|c|c|c|c|}
\hline PUBLICAÇÃO & $\begin{array}{c}\text { ANO DE } \\
\text { PUBLICAÇÃOO }\end{array}$ & $\begin{array}{c}\text { № DE } \\
\text { EXEMPLARES } \\
\text { CONSULTADOS }\end{array}$ & $\begin{array}{l}\text { № TOTAL DE } \\
\text { ARTIGOS } \\
\text { PUBLICADOS } \\
\text { NO PERÍODO }\end{array}$ & $\begin{array}{l}\text { № TOTAL DE } \\
\text { ARTIGOS DE } \\
\text { CONTABILIDADE } \\
\text { PUBLICADOS }\end{array}$ & $\begin{array}{c}\text { PARTICIPAÇÃO } \\
\text { DOS } \\
\text { ARTIGOS DE } \\
\text { CONTABILIDADE }\end{array}$ \\
\hline $\begin{array}{l}\text { PPE - Pesquisa e } \\
\text { Planejamento Econômico }\end{array}$ & $04 / 1990$ a $12 / 2001$ & 32 & 199 & 4 & $2,01 \%$ \\
\hline $\begin{array}{l}\text { RAC - Revista de } \\
\text { Administração Contemporânea }\end{array}$ & $04 / 1997$ a 12/ 2003 & 20 & 167 & 1 & $0,60 \%$ \\
\hline $\begin{array}{l}\text { RBE - Revista Brasileira } \\
\text { de Economia }\end{array}$ & $03 / 1990$ a $06 / 2003$ & 47 & 312 & 4 & $1,28 \%$ \\
\hline $\begin{array}{l}\text { RAP - Revista de } \\
\text { Administração Pública }\end{array}$ & $01 / 1990$ a $12 / 2003$ & 61 & 523 & 10 & $1,91 \%$ \\
\hline $\begin{array}{l}\text { RAE - Revista de } \\
\text { Administração de Empresas }\end{array}$ & $03 / 1990$ a $12 / 2003$ & 59 & 482 & 21 & $4,36 \%$ \\
\hline $\begin{array}{l}\text { RAUSP - Revista } \\
\text { de Administração }\end{array}$ & $03 / 1990$ a $12 / 2003$ & 50 & 354 & 20 & $5,65 \%$ \\
\hline Total & & 269 & 2.037 & 60 & $2,95 \%$ \\
\hline
\end{tabular}


pesquisador na utilização desse paradigma é medir 0 objeto de interesse da forma como ele existe no mundo (Denzin e Lincoln, 2000). 0 paradigma explicativo, que busca identificar os fatores que determinam ou contribuem para a ocorrência dos fenômenos, éo segundo mais utilizado. Chama a atenção a crescente adoção desse paradigma nos últimos anos pesquisados.
Adicionalmente, da totalidade dos artigos analisados, foram identificadas 75 estratégias de pesquisa, 0 que significa que al guns autores combinaram mais de uma estratégia. Os resultados do nível 2, referente à estratégia de pesquisa, foram: 33,3\% laboratório (25 artigos); $26,7 \%$ observação (20 artigos); $14,7 \%$ empírico-descritiva (11 artigos); $13,3 \%$ estudo de caso (10

Tabela 2 - Evolução anual do número de artigos publicados.

\begin{tabular}{|c|c|c|c|c|c|c|c|}
\hline ANO & PPE & RAC & RBE & RAP & RAE & RAUSP & TOTAL \\
\hline 1990 & 1 & NP & - & - & 3 & - & 4 \\
\hline 1991 & - & NP & - & - & - & 1 & 1 \\
\hline 1992 & - & NP & - & - & 2 & 1 & 3 \\
\hline 1993 & - & NP & - & 3 & - & - & 3 \\
\hline 1994 & - & NP & - & - & 4 & 1 & 5 \\
\hline 1995 & - & NP & - & 2 & 1 & - & 3 \\
\hline 1996 & - & NP & 1 & - & 1 & 2 & 4 \\
\hline 1997 & - & 1 & 1 & 1 & 3 & 2 & 8 \\
\hline 1998 & - & - & - & 1 & 1 & 1 & 3 \\
\hline 1999 & 2 & - & - & - & 3 & 1 & 6 \\
\hline 2000 & - & - & 1 & - & 1 & 1 & 3 \\
\hline 2001 & 1 & - & - & 3 & 1 & 4 & 9 \\
\hline 2002 & ND & - & 1 & - & - & 3 & 4 \\
\hline 2003 & ND & - & - & - & 1 & 3 & 4 \\
\hline Total & $\mathbf{4}$ & $\mathbf{1}$ & $\mathbf{4}$ & $\mathbf{1 0}$ & $\mathbf{2 1}$ & $\mathbf{2 0}$ & $\mathbf{6 0}$ \\
\hline Média & $\mathbf{0 , 2 8}$ & $\mathbf{0 , 1 4}$ & $\mathbf{0 , 2 8}$ & $\mathbf{0 , 7 1}$ & $\mathbf{1 , 5}$ & $\mathbf{1 , 4 3}$ & $\mathbf{4 , 3}$ \\
\hline
\end{tabular}

Obs.: ND = Não disponível; NP = Não publicado.

Tabela 3 - Número de artigos publicados por área.

\begin{tabular}{|l|c|c|c|c|c|c|c|c|}
\hline \multicolumn{1}{|c|}{ AREA DE CONTABILIDADE } & PPE & RAC & RBE & RAP & RAE & RAUSP & TOTAL & $\%$ \\
\hline Contabilidade de Custos & 1 & 1 & 4 & 1 & 7 & - & 14 & 23,3 \\
\hline Contabilidade Gerencial & - & - & - & - & 6 & 8 & 14 & 23,3 \\
\hline $\begin{array}{l}\text { Contabilidade e Mercados } \\
\text { de Capital }\end{array}$ & 1 & - & - & - & 2 & 7 & 10 & 16,7 \\
\hline Contabilidade Pública & 2 & - & - & 3 & - & - & 5 & 8,3 \\
\hline Contabilidade Tributária & - & - & - & 1 & 3 & 1 & 5 & 8,3 \\
\hline Orçamento & - & - & - & 4 & - & - & 4 & 6,7 \\
\hline Capital Intelectual & - & - & - & 1 & 1 & - & 2 & 3,3 \\
\hline Contabilidade Financeira & - & - & - & - & 1 & 1 & 2 & 3,3 \\
\hline Sistemas de Informação & - & - & - & - & - & 2 & 2 & 3,3 \\
\hline Aspectos Comportamentais & - & - & - & - & - & 1 & 1 & 1,7 \\
\hline Planejamento Financeiro & - & - & - & - & 1 & - & 1 & 1,7 \\
\hline Total & 4 & 1 & 4 & 10 & 21 & 20 & 60 & 100 \\
\hline
\end{tabular}


artigos); $10,7 \%$ pesquisa de campo ( 8 artigos); e, 1,3\% bibliográfica ( 1 artigo). Como estratégia de pesquisa se destaca o que se denomina laboratório, que consiste na construção de modelos e simulações matemáticas e estatísticas. Na maioria destes artigos, essa estratégia é utilizada conjuntamente com a da observação e a empírico-descritiva, principal mente em artigos relacionados à temática da contabilidade e de mercados de capital.

Quanto aos resultados da análise referente ao nível 3 - método de análise dos dados -, observou-se que em $40,9 \%$ dos artigos foi utilizada a análise expositiva ( 27 artigos); em 19,7\%, a análise comprobatória (13 artigos); em 16,7\%, a análise interpretativa (11 artigos); e em $13,6 \%$, a análise crítica ( 9 artigos). 0 menor percentual está relacionado às análises estatísticas, com 9,1\% dos casos. Outro aspecto a ser destacado, e que também está relacionado ao nível 1, é a falta de evidências de contribuição teórica em praticamente todos os artigos.

\section{Distribuição dos artigos de contabilidade nos periódicos}

Seguindo o mesmo critério de pesos estabelecido para as autorias na distribuição geográfica, constata-se que a maior participação é de autores vinculados à USP, com $28,3 \%$ da produção total, seguida pela FGV-SP e FGV-RJ, com uma participação de $15,8 \%$ e $11,6 \%$ respectivamente. É importante observar que essas três instituições são responsáveis por mais de $50 \%$ do total da produção anal isada, conforme a Tabela 4. Eventuais efeitos das políticas editoriais sobre o padrão das publicações não foram objeto de estudo neste artigo. Para conhecer esses efeitos na área de finanças, recomendam-se os estu dos de M itenko e Diamond (1994).

Os dados da Tabela 5 refletem uma característica dos periódicos analisados: são pou cos os trabal hos com mais de dois autores. Essa constatação difere do que se observa em eventos como o Enan pad, onde são comuns artigos com três ou quatro autores, conforme Leal, Oliveira e Soluri (2003) e Tonelli et al. (2003) salientaram em relação às áreas de finanças e recursos humanos, respectivamente.

Outro dado relevante é a grande concentração de autores que publicaram um único artigo. Do total de 77 autores, segundo o número de artigos em que 0 mesmo autor aparece como autor ou co-autor, verificou-se que 62 autores publicaram apenas um e 10 au-

Tabela 4 - Número de artigos publicados segundo o vínculo profissional dos autores.

\begin{tabular}{|l|c|c|c|c|c|c|c|c|}
\hline & PPE & RAC & RBE & RAP & RAE & RAUSP & TOTAL & $\%$ \\
\hline USP & 1,5 & - & - & 1,5 & 2,5 & 11,5 & 17,0 & 28,3 \\
\hline FGV-SP & - & - & - & 0,5 & 9,0 & - & 9,5 & 15,8 \\
\hline FGV-RJ & 1,0 & - & - & 5,0 & 1,0 & - & 7,0 & 11,6 \\
\hline UFRGS & - & - & - & - & 0,5 & 5,0 & 5,5 & 9,1 \\
\hline UFRJ & - & - & 1,0 & 0,5 & 2,5 & - & 4,0 & 6,6 \\
\hline UFSC & 1,0 & - & 1,0 & - & - & 1,0 & 3,0 & 5,0 \\
\hline Unesp & - & - & - & - & 1,5 & 0,5 & 2,0 & 3,3 \\
\hline University of Texas & - & - & - & - & 2,0 & - & 2,0 & 3,3 \\
\hline UFC & - & - & - & 1,0 & - & - & 1,0 & 1,6 \\
\hline PUC-SP & - & - & - & - & 1,0 & - & 1,0 & 1,6 \\
\hline PUC-RJ & - & - & - & 0,5 & - & 0,5 & 1,0 & 1,6 \\
\hline PUC-MG & - & - & - & 1,0 & - & - & 1,0 & 1,6 \\
\hline Alfenas & - & 1,0 & - & - & - & - & 1,0 & 1,6 \\
\hline Matanzas - Cuba & - & - & - & - & - & 1,0 & 1,0 & 1,6 \\
\hline Concepción - Chile & - & - & 1,0 & - & - & - & 1,0 & 1,6 \\
\hline PUC-RS & - & - & - & - & - & 0,5 & 0,5 & 0,8 \\
\hline University of Illinois & 0,5 & - & - & - & - & - & 0,5 & 0,8 \\
\hline University of Wisconsin & - & - & - & - & 0,3 & - & 0,3 & 0,5 \\
\hline Não definido & - & - & 1,0 & - & 0,7 & - & 1,7 & 2,7 \\
\hline Total & $\mathbf{4 , 0}$ & $\mathbf{1 , 0}$ & $\mathbf{4 , 0}$ & $\mathbf{1 0 , 0}$ & $\mathbf{2 1 , 0}$ & $\mathbf{2 0 , 0}$ & $\mathbf{6 0 , 0}$ & $\mathbf{1 0 0}, 0$ \\
\hline
\end{tabular}


tores publicaram dois artigos. Esse resultado sugere a falta de continuidade nas pesquisas ou na publicação dessa continuidade. Outra questão importante é que quatro autores publicaram três artigos e somente um autor publicou cinco artigos nos periódicos analisados, isto é, apenas cinco autores publicaram mais de dois artigos na área de contabilidade, e, dentro da amostra analisada, nenhum autor publicou mais do que cinco artigos. Esses resultados, quando comparados aos obtidos em áreas mais consolidadas do campo da Administração - de acordo com Leal, Oliveira e Soluri (2003) para o caso de finanças, e Tonelli et al. (2003) para o caso de recursos humanos - revelam que o nível de desenvolvimento teórico e de pesquisas empíricas na área de contabilidade publicada em periódicos nacionais " $A$ " é baixo.

\section{Análise comparativa da produtividade dos autores}

Pela Lei de Lotka - conforme descrita no item "Análise da produtividade dos autores" -, em uma amostra de 77 autores, a quantidade dos que possuem uma única publicação deveria ser 47 , o equivalente a $60,8 \%$ da amostra. Entretanto, o que se observa na pesquisa é que esse número foi de 62 ( $80,25 \%)$. Isso sugere que a produtividade nacional é inferior ao padrão internacional, se forem considerados os critérios de Lotka. $\mathrm{N}$ a Tabela 6 são comparados os dados observados e os padrões internacionais.

Leal, Oliveira e Soluri (2003) também constataram uma produtividade inferior aos padrões internacionais ao analisar a produtividade dos autores brasileiros na área de finanças. De acordo com os pesquisadores, $77,5 \%$ dos autores da amostra anal isada apresentaram apenas uma publicação. Uma comparação entre os dados da amostra e os resul tados constatados por Leal, Oliveira e Soluri (2003) é feita a seguir.

Enquanto na presente pesquisa se constatou que $80,52 \%$ dos artigos da área são trabalhos únicos de um autor, Leal, Oliveira e Soluri (2003) constataram 77,5\% na área de finanças; para dois artigos por autor, foram observados $12,98 \%$ contra $11,50 \%$; para três artigos foram observados $5,19 \%$ contra $5,5 \%$; para quatro artigos não houve ocorrência neste estudo, enquanto Leal, Oliveira e Soluri constataram 1,9\%; para cinco artigos foram observados 1,3\% contra 1,2\%; e para mais de cinco artigos, novamente não houve ocorrência neste estudo, enquanto Leal, Oliveira e Soluri constataram $2,4 \%$.

Tabela 5 - Número de autores por artigo.

\begin{tabular}{|l|c|c|c|c|r|r|r|r|r|}
\hline № DE AUTORES & PPE & RAC & RBE & RAP & RAE & RAUSP & TOTAL & $\%$ \\
\hline 1 & 2 & 1 & 1 & 6 & 11 & 7 & 28 & 46,67 \\
\hline 2 & 2 & - & 3 & 3 & 6 & 11 & 25 & 41,66 \\
\hline 3 & - & - & - & 1 & 4 & 2 & 7 & 11,67 \\
\hline Total & $\mathbf{4}$ & $\mathbf{1}$ & $\mathbf{4}$ & $\mathbf{1 0}$ & $\mathbf{2 1}$ & $\mathbf{2 0}$ & $\mathbf{6 0}$ & $\mathbf{1 0 0}$ \\
\hline
\end{tabular}

Tabela 6 - Comparação da produtividade entre os dados da amostra em questão e o padrão internacional.

\begin{tabular}{|l|r|r|r|r|}
\hline \multirow{2}{*}{$N$ DE ARTIGOS } & \multicolumn{4}{|c|}{ N.. DE AUTORES } \\
\hline POR AUTOR & DADOS OBSERVADOS & $\%$ & PADRÃO INTERNACIONAL & $\%$ \\
\hline 1 & 62 & 80,52 & 47 & 60,80 \\
\hline 2 & 10 & 12,98 & 12 & 15,20 \\
\hline 3 & 4 & 5,19 & 5 & 6,80 \\
\hline 4 & 0 & 0,00 & 3 & 3,80 \\
\hline 5 & 1 & 1,30 & 2 & 2,43 \\
\hline 6 & - & & 1 & 1,69 \\
\hline 7 & - & - & 1 & 1,24 \\
\hline+7 & 7 & & 6 & 8,04 \\
\hline Total & 77 & & & 100 \\
\hline
\end{tabular}


A análise da tabela 6 mostra que os autores da área de finanças possuem uma produtividade maior se comparada à área de contabilidade. É importante observar que é significativo o percentual de autores da área de finanças com mais que cinco artigos publicados. Na Tabela 7 são expostos os autores com 0 maior número de artigos publicados na área de contabilidade.

Ainda em relação ao autores, considerou-se o vínculo institucional citado na época da publicação do artigo. 0 que pode ser percebido é que a grande maioria desses pesquisadores não estava vinculada diretamente a programas de pós-graduação em contabilidade na época da publicação, o que sugere a necessidade de incremento nas pesquisas pelos próprios programas de pós-graduação da área.

Em outro estudo, Chung, Cox e Pak (1992) analisaram a produtividade de autores, na área contábil, que publicaram em 14 periódicos de língua inglesa selecionados entre 1968 e 1988. Os resultados obtidos revelam uma concentração um pouco maior do que a prevista pela Lei de Lotka para autores com mais do que uma publicação.

\section{A generalização da Lei de Lotka}

Da mesma forma que Chung, Cox e Pak (1992) e Leal, Oliveira e Soluri (2003), apresentamos a seguir uma estimativa para o coeficiente $c$ da Lei de Lotka genéri- ca, expressa por:

$$
\alpha_{n}=\alpha_{1} \cdot \frac{1}{n^{c}}
$$

Conforme já salientado no item "Análise da produtividade dos autores", o coeficiente c pode ser estimado pela técnica da regressão linear. Para a amostra em questão, excluindo 0 evento $n=4$, para 0 qual não temos nenhuma ocorrência, o coeficiente $c$ calculado foi 2,54. 0 coeficiente de correlação entre as variáveis em questão foi 0,9995 , o que corresponde a um grau de confiança de $99 \%$. O coeficiente de determinação $\mathrm{R}^{2}$ resultou em 0,9987, o que representa um alto poder explicativo do modelo.

Com esse modelo, pode-se concluir que o número de autores com mais de uma publicação na amostra em questão pode ser estimado a partir da equação abaixo:

$$
\alpha_{n}=\alpha_{1} \cdot \frac{1}{n^{2,54}}
$$

Cabe observar que esse coeficiente c de 2,54 é maior do que o padrão de Lotka e do que os calculados por Chung, Cox e Pak (1992), e Leal, Oliveira e Soluri (2003). I sso significa que o número de autores com uma única publicação na amostra é maior do que em

\begin{tabular}{|c|c|c|c|c|c|c|c|c|}
\hline NOME DO AUTOR & INSTITUIÇÄO & TOTAL & PPE & $\mathrm{RAC}$ & RBE & RAP & RAE & RAUSP \\
\hline Procianoy, J. L. & UFGRS & 5 & & & & & 1 & 4 \\
\hline Dias, I. P. & FGV-SP & 3 & & & & & 3 & \\
\hline Limberg, S. T. & U. of Texas & 3 & & & & & 3 & \\
\hline Robison, J. R. & U. of Texas & 3 & & & & & 3 & \\
\hline Famá, $R$. & USP & 3 & & & & & 1 & 2 \\
\hline Rocha, F. & USP & 2 & 2 & & & & & \\
\hline Sanches, O. M. & FGV-RJ & 2 & & & & 2 & & \\
\hline Marques, J. A. V. C. & UFRJ & 2 & & & & & 2 & \\
\hline Fusco, J. P. A. & Unesp & 2 & & & & & 1 & 1 \\
\hline Khoury, C. Y. & FGV-SP & 2 & & & & & 2 & \\
\hline Frezatti, F. & USP & 2 & & & & & & 2 \\
\hline Sanvicente, A. Z. & USP & 2 & & & & & 1 & 1 \\
\hline Ancelevicz, J. & FGV-SP & 2 & & & & & 2 & \\
\hline Christians, R. L. & - & 2 & & & & & 2 & \\
\hline Leite, $H$. de P. & FGV-SP & 2 & & & & & 2 & \\
\hline
\end{tabular}

Tabela 7 - Autores que publicaram mais de um artigo nos periódicos selecionados. 
todas as outras examinadas pel os pesquisadores anteriormente citados. U ma síntese desses dados é mostrada na Tabela 8.

Os dados da Tabela 7 sugerem que os autores da amostra em estudo apresentam uma produtividade bem inferior à dos seus pares que publicam em periódicos de língua inglesa, e abaixo dos autores da área de finanças que publicam em periódicos nacionais. Esses resultados não são conclusivos em função da diversidade das amostras analisadas, mas são indicativos das características dos trabalhos acadêmicos na área da contabilidade.

\section{CONSIDERAÇÕES FINAIS}

Este artigo procurou contribuir para um melhor entendimento do estágio atual da produção acadêmica da área da contabilidade por meio da análise bibliométrica. A pesar da inexistência de estudos específicos no Brasil que permitam definir um padrão sobre a média de publicação por autor, os resultados deste estudo mostram que a participação da produção em contabilidade é baixa, sendo de apenas 2,95\% em relação ao total de artigos publicados no período pesquisado. Este estudo é rel evante porque mostra que os autores nacionais na área contábil apresentam uma produção menor em relação à produção internacional, e também inferior à dos seus pares em outras áreas da Administração, como a de finanças. Outro fator é a concentração da pesquisa publicada, com poucos autores com um número relevante de artigos, e muitos não vinculados diretamente a programas de pós-graduação em contabilidade.

Apesar de se limitar a um grupo de periódicos, este estudo gera evidências de que o crescimento da produção ainda é baixo em relação às expectativas da comunidade acadêmica brasileira de contabilidade e da própria sociedade. Ressalta-se a abordagem metodológica, em que as evidências sugerem a baixa contribuição teórica dos artigos, al ém da carência de es- tudos estruturados na sua abordagem quantitativa e qualitativa. Assim, é importante a realização de mais estudos sobre o tema, bem como suscitar discussões na comunidade contábil sobre as causas de tal deficiência.

Em relação às limitações dessa pesquisa, há duas questões que precisam ser ressaltadas. Primeiro, o período de abrangência é de 1990 a 2003, não levando em conta, portanto, a produção anterior à década de 1990; segundo, o critério de escolha dos periódicos analisados, ao considerar apenas os que possuíam 0 conceito " $A$ " pela Capes durante o período de abrangência do estudo, não levou em conta os periódicos de conceito " $B$ " $e$ " $C$ ", bem como os anais de congressos como o Enanpad.

Para futuras pesquisas sugere-se a aplicação de outros recursos de bibliometria, como as Leis de Zipf e de Bradford mencionadas no início deste artigo, e a análise de conteúdo dos artigos de uma temática específica, dentre as abarcadas no presente estudo. Outro aspecto interessante a ser pesquisado é referente à linha editorial dos periódicos anal isados quanto aos critérios de aceitação de textos de contabilidade. Por último, cabe ressal tar a importância de discussões sobre as causas efetivas do reduzido volume de publicações e a possível existência de círculo vicioso e de seu efeito sobre a falta de recursos para a pesquisa.

\section{REFERÊNCIAS BIBLIOGRÁFICAS}

ARKADER, R. A pesquisa científica em gerência de operações no Brasil. Revista de Administração de Empresas, v. 43, n. 1, p. 70-80, 2003.

BRICKER, R. An empirical investigation of the structure of accounting research. Journal of Accounting Research, v. 27, n. 2, p. 246-262, 1989.

CALDAS, M. P.; TINOCO, T. Pesquisa em gestão de recursos humanos nos anos 1990: um estudo bibliométrico. Revista de Administração de Empresas, v. 44, n. 3, p. 100-114, 2004.

Tabela 8 - Coeficiente $c$.

\begin{tabular}{|l|c|c|c|c|}
\hline \multicolumn{1}{|c|}{ ESTUDOS } & $\begin{array}{c}\text { DADOS DE } \\
\text { CHUNG, COXE PAK (1992) }\end{array}$ & $\begin{array}{c}\text { PADRÃO } \\
\text { LEI DE LOTKA }\end{array}$ & $\begin{array}{c}\text { LEAL, OLIVEIRA E } \\
\text { SOLURI (2003) }\end{array}$ & $\begin{array}{r}\text { DADOS DA } \\
\text { AM OS TRA }\end{array}$ \\
\hline C & 1,87 & 2,00 & 2,44 & 2,54 \\
\hline $\begin{array}{l}\text { \% de autores com um único } \\
\text { artigo publicado }\end{array}$ & 57,70 & 60,80 & 77,50 & 80,52 \\
\hline
\end{tabular}


CARSON, P. et al. A historical perspective on fad adoption and abandonment. Journal of M anagement History, v. 5, n. 6, p. 320, 1999.

CHEN, Y.; CHONG, P. P; TONG, M. Y. The Simon-Yule approach to bibliometric modeling. Information Processing $\&$ Management, v. 30, n. 4, p. 535-56, 1994.

CHUNG, K. H.; COX, R. A. K. Patterns of productivity in the finance literature: a study of the bibliometric distributions. The Journal of Finance, v. 45, n. 1, p. 301-309, 1990.

CHUNG, K. H.; COX, R. A. K.; PAK, H. S. Patterns of research output in the accounting literature: a study of the bibliometric distributions. Abacus, v. 28, n. 2, p. 168-185, 1992.

DA SILVA, J. A. Cientometria: a métrica da ciência. Paidéia: Cadernos de Psicologia e Educação, Ribeirão Preto, v. 11, n. 20, p. 5-10, 2001.

DENZIN, N.; LINCOLN, Y. (Eds.). Handbook of Qualitative Research. Thousand Oaks, CA: Sage Publication, 2000.

FIN DLAY, A.; SPARKS, L. European retail journals: a bibliometric analysis. International Journal of Retail \& Distribution Management, v. 30, n. 8, p. 373-382, 2002.

FREZATTI, F. Análise dos traços de tendências de uma amostra das revistas científicas da área de contabilidade publicadas na língua inglesa. Caderno de Estudos, São Paulo, v. 13, n. 24, p. 50-78, 2000.

GARCIA-CASTRILLO, P.; MONTAÑÉS, A.; SANZ-GRACIA, F. A worldwide assessment of scientific production in economics (1992-1997). Applied Economics, v. 34, p. 1453-1475, 2002.

HOPPEN, N. L.; MEIRELLES, F. S. Sistemas de informação: a pesquisa científica brasileira entre 1990 e 2003. Revista de Administração de Empresas, v. 45, n. 1, p. 24-35, 2005.

LEAL, R. P. C; OLIVEIRA, J.; SO LURI, A. F. Perfil da pesquisa em finanças no Brasil. Revista de Administração de Empresas, v. 43, n. 1, p. 91-103, 2003.

LEYDESDORFF, L. The Challenge of Scientometrics: The Development, Measurement, and Organization of Scientific Communications. Universal Publishers, 2001.

MACHADO-DA-SILVA, C. L.; CUNHA, V.; AMBONI, N. Organizações: 0 estado da arte da produção acadêmica. In: ENCONTRO NACIONAL DA ASSOCIAÇÃO NACIONAL DOS PROGRAMAS DE PÓS-GRADUAÇÃO EM ADMINISTRAÇÃO, 14., 1990, Florianópolis. Anais. Florianópolis: ANPAD, p. 11-28, 1990.

MACIAS-CHAPULA, C. A. 0 papel da informetria e da cienciometria e sua perspectiva nacional e internacional. Ciência da informação, v. 27, n. 2 , p. 134-140, 1998. Disponível em: <http://www.unicamp.br/bc/ RelSBU_online/glossario/glossario.htm>. Acesso em: 12 abr. 2004.
MARTINS, G. Considerações sobre os doze anos do caderno de estudos: divulgação de trabalho. Revista Contabilidade \& Finanças - USP, n. 30, p. 81-88, 2002.

MCMILLAN, G. S.; HICKS, D. Science and corporate strategy: a bibliometric update of H ounshell and Smith. Technology Analysis \& Strategic Management, v. 13, n. 4, p. 497-505, 2001.

MCMILLAN, G. S.; HAMILTON, R. D. Using bibliometrics to measure firm knowledge: an analysis of the US pharmaceutical industry. Technology Analysis \& Strategic Management, v. 12, n. 4, p. 465-475, 2000.

MITENKO, G. R.; DIAMOND, J. M. Publication policies and practices of major journals: a survey of editors. Financial Practice and Education, v. 4, n. 2, p. 88-93, 1994.

OLIVEIRA, M. Análise dos periódicos brasileiros de contabilidade. Revista Contabilidade \& Finanças - USP, n. 29, p. 68-86, 2002.

OTCHERE, I. Accounting and Finance at forty: a retrospective evaluation. Accounting \& Finance, v. 43, n. 2, p. 211-231, 2003.

PARK, S. H.; GORDON, M. E. Publication records and tenure decisions in the field of strategic management. Strategic Management Journal, v. 17, n. 2, p. 109-129, 1996.

PILKINGTON, A.; LISTON-HEYES, C. Is production and operations management a discipline? A citation/co-citation study. International Journal of Operations \& Production Management, v. 19, n. 1, p. 7-20, 1999.

QUALIS - Sistema de Periódicos, Anais e Revistas, Capes. Disponível em: ४tttp://qualis.capes.gov.br/>. Acesso em: Set. 2004.

RICCIO, E. L.; CARASTAN, J.; SAKATA, M. C. G. Accounting research in Brazilian universities: 1962-1999. Caderno de Estudos, São Paulo, Fipecafi, n. 22, 1999.

SELLTIZ, C.; COOK S. W.; WRIGHTSMAN, L. S. Métodos de pesquisa nas relações sociais. São Paulo: EPU, 1987

SHIELDS, M. Research in management accounting by North Americans in the 1990s. Journal of Management Accounting Research, v. 9, p. 3-60, 1997.

SOUZA, F. As possibilidades pedagógicas no ensino de metodologia da pesquisa científica e em ciência da informação e os objetos deste campo científico: aproximações durkheimianas. Revista Eletrônica de Biblioteconomia e Ciência da Informação, Florianópolis, n. 16, p. 20-40, 2003.

SPELL, C. S. Where do management fashions come from, and how long do they stay? Journal of Management History, v. 5, n. 6, p. 334, 1999. 
TONELLI, M. J. et al. Produção acadêmica em recursos humanos no Brasil: 1991-2000. Revista de Administração de Empresas, v. 43, n. 1, p. 104122, 2003.

VANTI, N. A. P. Da bibliometria à webometria: uma exploração conceitual dos mecanismos utilizados para medir o registro da informação e a difusão do conhecimento. Revista Ciência da Informação, v. 31, n. 2, p. 152162,2002

VERBEEK, A. et al. Measuring progress and evolution in science and technology - I: The multiple uses of bibliometric indicators. International Journal of Management Reviews, v. 4, n. 2, p. 179-211, 2002.
VERGARA, S.; PINTO, M. C. S. N acionalidade das referências teóricas em análise organizacional: um estudo das nacionalidades dos autores referenciados na literatura brasileira. In: ENCONTRO NACIONAL DE ESTUDOS ORGANIZACIONAIS, 24., 2000, Curitiba. Anais. Curitiba: ANPAD, 2000.

VIEIRA, F. G. D. Por quem os sinos dobram? Uma análise da publicação científica na área de marketing do Enanpad. In: ENCONTRO NACIONAL DE ESTUDOSORGANIZACIONAIS, 22., 1998, Foz do I guaçu. Anais. Foz do Iguaçu: AN PAD, 1998.

ZEFF, S. A. A study of academic research journals in accounting. Accounting Horizons, v. 10, n. 3, p. 158-177, 1996.

\section{Artigo recebido em 10.08.2004. Aprovado em 25.02.2005.}

\section{Ricardo Lopes Cardoso}

Professor de Contabilidade e Controladoria da PUC-Campinas. Doutorando em Controladoria e Contabilidade na FEA-USP.

Interesses de pesquisa nas áreas de contabilidade, cientometria, métricas em contabilidade e contabilidade comportamental.

E-mail: ricardo@puc-campinas.edu.br

Endereço: Estrada Municipal 401, 70, Cond. Terras do Oriente, Capuava, Valinhos - SP, 13272-181.

\section{Octávio Ribeiro de Mendonça Neto}

Professor de Finanças da UMESP. Doutorando em Controladoria e Contabilidade na FEA-USP.

Interesses de pesquisa nas áreas de contabilidade, finanças e cientometria.

E-mail: octaviomendonca@aol.com

Endereço: Alameda dos Guaiases, 659, São Paulo - SP, 04079-011.

\section{Edson Luiz Riccio}

Professor da FEA-USP. Doutor em contabilidade e controladoria.

Interesses de pesquisa nas áreas de contabilidade, sistemas de informações e cientometria.

E-mail: elriccio@usp.br

Endereço: Av. Prof. Luciano Gual berto, 908, FEA 3, Bairro Cidade Universitária, São Paulo - SP, 05508-900.

\section{Marici Cristine Gramacho Sakata}

Pesquisadora do Laboratório de Tecnologia e Sistemas de Informação (TECSI) da FEA-USP. Doutoranda em Comunicação na ECA-USP.

Interesses de pesquisa nas áreas de contabilidade, comunicação e cientometria.

E-mail: mcsakata@usp.br

Endereço: Av. Prof. Luciano Gualberto, 908, FEA 3, Cidade Universitária, São Paulo - SP, 05508-900. 